\title{
Las manillas como tejido del pensamiento del pueblo Inga ${ }^{1}$
}

\author{
Francisco Andrés Muñoz Burbano \\ Resguardo INGA Aponte-Nariño, Colombia
}

Recepción: 20/04/2020

Evaluación: 27/04/2020

Aprobación: 11/05/2020

Artículo de Investigación-Científica

DOI: https://doi.org/10.22267/rhec.202424.77

\section{Resumen}

Esta propuesta de investigación aborda la importancia de la cultura en la educación para la juventud y existencia de los pueblos indígenas; desde esta perspectiva, las expresiones simbólicas, que desde el tejido se han venido trabajando por las artesanas durante varias generaciones, tienen un contenido esencial para complementar las demás áreas de conocimiento que esta comunidad necesita fortalecer; este planteamiento forma parte de una propuesta de investigación denominada "Iuiay kamsaykug Tantalli" Las Manillas como Tejido del Pensamiento del Pueblo Inga. El aporte de esta investigación consistió en entregar una propuesta educativa enfocada en la simbología indígena inga, para permitir con ello a los docentes estructurar nuevos contenidos desde sus contextos, para trabajarlos con los temas pedagógicos que comúnmente se imparten a los estudiantes, con un fortalecimiento de la construcción de un proyecto de educación propia, muy necesario de implementar en esta comunidad.

Por otra parte, se observó en este proceso la importancia de reivindicar los conocimientos guardados en estos tejidos, interpretados desde el punto de vista de sus propios autores, quienes son herederos de esta tradición antigua, con descripción de temáticas muy necesarias para estudiar y rescatar en los contextos educativos actuales, lo que se convierte en una

1 Este artículo es resultado y forma parte del proyecto de investigación: "IUIAY KAMSAYKUG TANTALLI Las manillas como tejido del pensamiento del pueblo Inga".

2 Investigador independiente. Resguardo INGA Aponte-Nariño, Colombia. Docente tutor del programa "Todos a Aprender". Magister en educación. Correo electrónico: paocho811@hotmail.com. 
alternativa que contribuye tanto a sistematizar como a salvaguardar los valores e identidad de esta comunidad, mediante el intercambio de saberes $\mathrm{y}$, a la vez, retornar los conocimientos propios de estas comunidades, con promoción de un vínculo con otras áreas del conocimiento pedagógico.

Palabras clave: educación; escritura; indígenas; símbolos; tejidos.

\title{
Bracelets as a representation of the Inga community way of thinking
}

\begin{abstract}
This research proposal addresses the importance of culture in education for the youth and the existence of indigenous peoples; from this perspective, the symbolic expressions that have been worked on by the artisans for several generations have an essential content to complement the other areas of knowledge that this community needs to strengthen; this approach is part of a research proposal called "Iuiay kamsaykug Tantalli" Las Manillas como Tejido del Pensamiento del Pueblo Inga (Bracelets as a Representation of the Inga community way of thinking). The contribution of this research consisted in delivering an educational proposal focused on Inga indigenous symbolism, in order to allow teachers to structure new content from their contexts, to work with the pedagogical themes that are commonly taught to students, with a strengthening of the construction of an own education project which is very necessary to implement in this community.

On the other hand, it was observed in this process the importance of claiming the knowledge kept in these textiles, interpreted from the point of view of their own authors, who are heirs of this ancient tradition, with description of very necessary topics to study and rescue in the current educational contexts, which becomes an alternative that contributes both to systematize and safeguard the values and identity of this community, through the exchange of knowledge and, at the same time, return to the knowledge of these communities, enhancing the link with other areas of pedagogical knowledge.
\end{abstract}

Keywords: education; writing; Inga community; symbols; weaving. 


\section{Pulceiras como tecido do pensamento do povo Inga}

\section{Resumo}

Esta proposta de pesquisa aborda a importância da cultura na educação da juventude e na existência dos povos indígenas; Nessa perspectiva, as expressões simbólicas, nas quais as artesãs trabalham há várias gerações, têm um conteúdo essencial para complementar as demais áreas do conhecimento que esta comunidade precisa fortalecer; Esta abordagem faz parte de uma proposta de pesquisa chamada "Iuiay kamsaykug Tantalli" As Pulceiras como um tecido de pensamento do povo Inga. A contribuição desta pesquisa consistiu na entrega de uma proposta pedagógica voltada para a simbologia indígena Inga, de forma a permitir aos professores estruturar novos conteúdos a partir de seus contextos, trabalhar com as temáticas pedagógicas comumente oferecidas aos alunos, com um fortalecimento da a construção de um projeto de educação próprio, muito necessário para implementar nesta comunidade.

Por outro lado, observou-se neste processo a importância de reivindicar o conhecimento armazenado nesses tecidos, interpretado do ponto de vista de seus próprios autores, herdeiros dessa tradição antiga, com a descrição de temas muito necessários para estudar e resgatar em. contextos educacionais atuais, que se torna uma alternativa que contribui tanto para sistematizar e salvaguardar os valores e a identidade desta comunidade, através da troca de saberes e, ao mesmo tempo, devolver o conhecimento dessas comunidades, com promoção de um vínculo com outras áreas do conhecimento pedagógico.

Palavras-chave: educação; escrita; indígena; símbolos; tecidos.

\section{Introducción}

Este artículo es el resultado de la investigación realizada en el Resguardo Inga de Aponte, ubicado en el Municipio del Tablón de Gómez, al nororiente del Departamento de Nariño. La investigación tuvo como objetivo conocer la sabiduría inmersa en los símbolos presentes en las manillas Inga, dado 
que no se ha trabajado en salvaguardar este patrimonio cultural de esta comunidad. Se buscó desarrollar una propuesta educativa en la Institución Educativa del Resguardo Inga de Aponte, enfocada en el fortalecimiento de la educación propia, que permite construir, conjuntamente con los docentes, un proyecto pedagógico articulado a la información obtenida en esta investigación desde el ámbito cultural, con los actuales contenidos educativos dentro del plan de estudios.

Por ello, se trabajó con uno de los referentes culturales más importantes para el Inga, en este caso los símbolos, debido al aporte que este tema podría ofrecer en materia educativa e investigativa, sobre todo en esta institución agropecuaria indígena. Los símbolos Inga son un tipo de textos, plasmados en los tejidos como las manillas, que describen contenidos de gran importancia para esta comunidad desde los aspectos astrológicos, biológicos, matemáticos, botánicos y culturales.

Por lo tanto, serían varias las temáticas que los docentes podrían trabajar en el aula de clase, ayudando así a complementarlas con otras áreas del conocimiento que se imparten en esta institución educativa, entre las cuales podríamos destacar: lenguaje, matemáticas, ciencias naturales, ciencias sociales, educación artística, historia, astronomía, agricultura, medicina tradicional, identidad, cultura y espiritualidad.

Estos contenidos surgieron a partir de esta investigación, para abordarlos desde el ámbito educativo, porque son importantes elementos de la visión del mundo del inga, como un rasgo característico de su historia e identidad cultural. Este aspecto permitió trabajar con los docentes estrategias pedagógicas, con unos lineamientos claros en los contenidos de educación propia; de esta forma, cumplir con las necesidades requeridas a fortalecer las potencialidades de estas poblaciones, donde las expresiones culturales fomentan su reconocimiento y desarrollo.

Esta investigación se llevó a cabo con participación de tejedoras de la comunidad inga residentes en Aponte, Departamento de Nariño. Un grupo de mujeres sabedoras de estos conocimientos simbólicos, vinculadas a los procesos textiles desde épocas remotas con otras comunidades indígenas; aunque no necesariamente trabajan con las manillas, han formado parte desde esa herencia histórica, en la cual el Resguardo inga de Aponte lleva más de 500 años de fundación y sus artesanas han trabajado la simbología durante generaciones, por lo que ellas serían las más indicadas para guiar la lectura e interpretación de las manillas desde sus símbolos más representativos. 
Por último, se dio a conocer este proceso a la Institución Educativa, para desarrollar un análisis, en conjunto con la comunidad educativa, sobre la importancia de incluir y fortalecer estos contenidos culturales, dentro de las áreas que comúnmente se venían trabajando en las aulas de clase, por lo que se recomendó una transversalidad de los saberes desde estos nuevos conocimientos, para utilizar este importante material como un complemento a las demás temáticas académicas, para contribuir en el proceso de consolidación del proyecto de educación propia en esta comunidad.

\section{Problema de investigación}

La investigación buscó responder a: ¿Qué aspectos simbólicos de la cultura Inga se encuentran presentes en los tejidos de las manillas y son importantes para complementar el proyecto educativo del Instituto Agropecuario Inga de Aponte?, para ello el objetivo principal fue analizar y organizar los aspectos simbólicos de la cultura inga que se encuentran presentes en los tejidos de las manillas y son de importancia para complementar el Proyecto Educativo del Instituto agropecuario Inga de Aponte; para ello, se planteó: analizar el simbolismo presente en las manillas de la cultura inga, organizar los contenidos culturales y simbólicos de las manillas Inga y diseñar una propuesta con base en los contenidos culturales y simbólicos que complementen el proyecto educativo del Instituto Agropecuario Inga de Aponte.

\section{Metodología}

La perspectiva metodológica de la investigación se desarrolló desde el paradigma cualitativo, lo que permite realizar un acercamiento de orden interpretativo al objeto de estudio en sus ambientes naturales, para conocer esa realidad con base en los significados de los símbolos en los tejidos de las manillas, que las personas les otorgan desde la experiencia humana y cultural. En ese sentido, desde la orientación de este paradigma, la investigación, en primer lugar, tuvo en cuenta que:

Las maneras de concebir la naturaleza del conocimiento y de la realidad se fundamentan en la existencia de un sujeto cognoscente, el cual está influido por una 
cultura y unas relaciones sociales particulares, que hacen que la realidad epistémica dependa para su definición, comprensión y análisis, del conocimiento de las formas de percibir, pensar, sentir y actuar, propias de esos sujetos cognoscentes. (Sandoval, 2002, p. 28)

Por ello, se trabajó directamente con artesanas, quienes ayudaron a interpretar la información recolectada, con utilización, como método, de la etnografía educativa, para comprender los procesos descriptivos de esta información, con un análisis de datos que incluye una interpretación de significados y funciones de las acciones humanas.

Se escogió una muestra de 30 manillas, que se destacan en la comunidad inga por sus representaciones simbólicas y significados dentro de su cosmovisión; las manillas las han suministrado las artesanas y artesanos más importantes de este pueblo ancestral, entre ellos es destacado el trabajo de Daysi Mavisoy, Ayda Quinchua, Mary Luz Quinchua y Cristóbal Córdoba. Ellos son representantes del Cabildo de la mujer y el Cabildo cultura en el Resguardo inga de Aponte, Departamento de Nariño.

$\mathrm{Al}$ tener en cuenta el tipo de investigación empleado en este proyecto, se utilizó, como técnica de recolección de información, la entrevista abierta y semiestructurada y la observación participante; estas técnicas organizaron la recolección de datos a partir del estudio de estos tejidos y cómo, desde la explicación de sus figuras, colores y símbolos, se logra desarrollar un análisis que nos dé una solución al problema planteado en esta investigación.

\section{Resultados}

Dentro de los hallazgos obtenidos en esta investigación se pueden mencionar:

Los símbolos estudiados en esta investigación, en total, corresponden a treinta (30) símbolos de la muestra, los cuales se organizaron en seis grupos:

1. Astrología: estos símbolos, figuras y colores son representaciones de constelaciones, planetas, estrellas y calendarios.

2. Plantas sagradas: se han identificado algunos elementos vegetales, los cuales son representativos desde su ancestralidad y cosmovisión. 
3. Animales: hay un gran número de animales, que son, desde el ámbito de la interpretación de esta comunidad indígena, una representación de la fuerza y poder espiritual, para convertirse en entes protectores e impartidores de conocimientos.

4. Seres espirituales: forman parte de la misma comunidad, pero están en un plano dimensional diferente, que no es físico, y se encargan de proteger y mantener el orden y los conocimientos en las comunidades.

5. Territorio: forma parte de todo el pensamiento y sentimiento del indígena; representa el origen y el hogar de donde surge todo.

6. Fertilidad: es fundamental en el pensamiento andino y se basa en la dualidad enfocada a la parte femenina, que se representa y conceptualiza desde la naturaleza como entidad creadora.

La comprensión e interpretación de la simbología impresa en las manillas se obtuvo a partir de tres lecturas:

1) Lectura del tejido chumbe inga, por parte de las artesanas, que es una prenda utilizada como faja.

2) Lectura de las visiones hechas por los taitas, resultado del ritual en el consumo de la planta sagrada (yagé).

3) Lectura dada durante las Mingas de Pensamiento con las artesanas, mediante reuniones periódicas y colectivas, donde se tratan temas socioculturales en el Resguardo; el objetivo es compartir experiencias.

Los símbolos utilizados por las artesanas, correspondientes a todos estos procesos, son estos:

En el primer grupo, denominado Astrología, se plasman ocho símbolos relacionados con este tema, tal como se muestra en la Figura 1: 


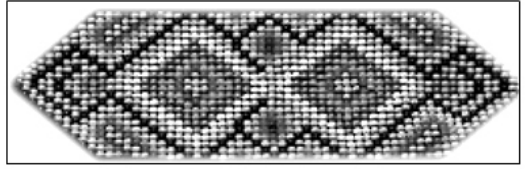

Símbolo 1. Chaska Nauimurukuna (Ojos de Estrella)

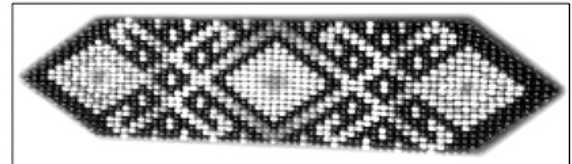

Símbolo 2. Uigsa Ñavi (Ojos de Vientre)

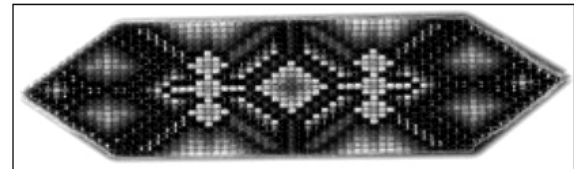

Símbolo 3. Chaska (Estrella)

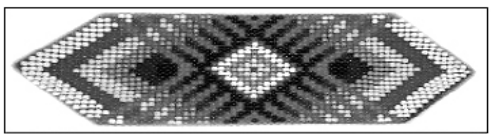

Símbolo 4. Inti (Sol)

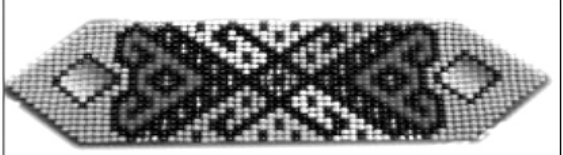

Símbolo 5. Suyukuna (Lugar)

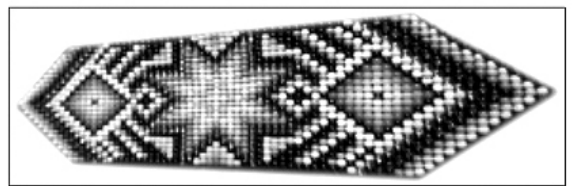

Símbolo 6. Estrella de ocho puntas

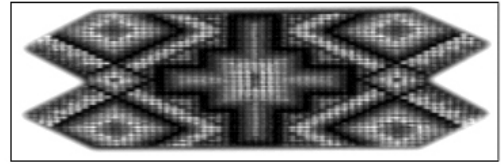

Símbolo 7. Chacana

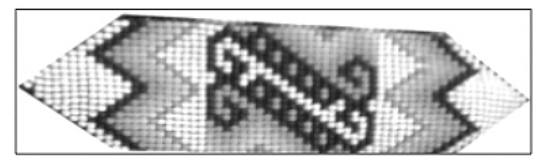

Símbolo 8. Churo

Figura 1. Grupo Astrología

Fuente: esta investigación.

Estos símbolos se refieren a las estrellas, la constelación de Orión, la estrella Sirio, el astro solar, los recorridos de los equinoccios y solsticios que marcan en el cielo durante el año dos puntos de referencia y representan una $\mathrm{x}$, la estrella de ocho puntas, la chacana y el churo cósmico. Todos estos conceptos nos presentan un conocimiento astronómico y geométrico matemático llevado al ámbito espiritual, como un elemento de asociación al propio origen estelar de su mito.

En el Grupo dos, denominado Plantas Sagradas, se plasman tres símbolos relacionados con este tema, como se ve en la Figura 2: 


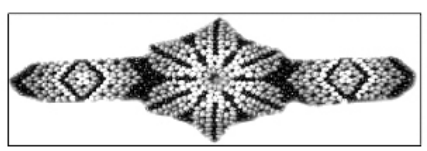

1.Tujtu (Flor)

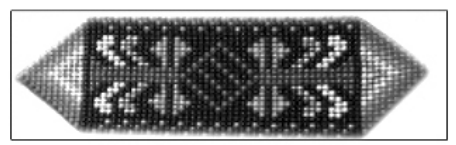

2. Sarra (Maíz)

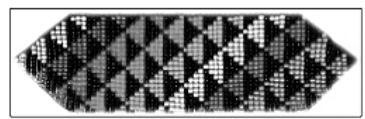

3.Ambihuasca

(Ayahuaska, Yagé)

Figura 2. Grupo Plantas Sagradas

Fuente: esta investigación.

Estos símbolos nos narran el profundo agradecimiento y amor que los indígenas tienen a su pachamama (Madre Tierra); por lo tanto, estos elementos se utilizan en celebraciones, curaciones y retiros espirituales, como una ofrenda solemne a los ancestros.

En el Grupo tres, denominado Animales Sagrados, se plasman ocho símbolos relacionados con este tema:

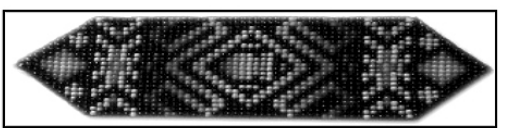

Mojojoy (Rana)

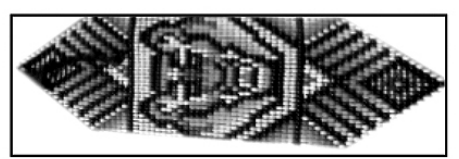

Jaguar

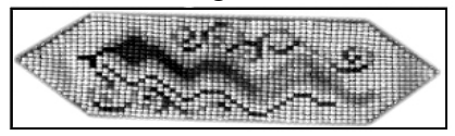

Serpiente

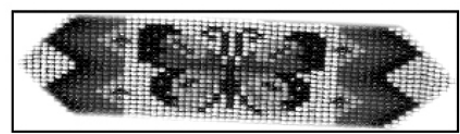

Mariposa

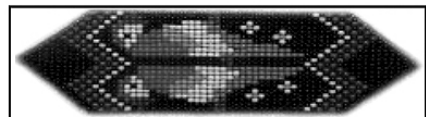

Guacamayas

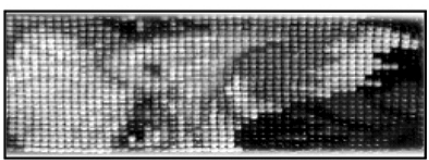

Condor

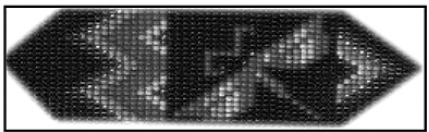

Libélula

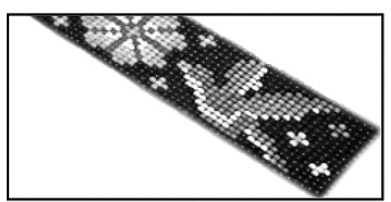

Kindi (Colibrí)

Figura 3. Grupo Animales Sagrados

Fuente: esta investigación 
Estos símbolos nos permiten adentrarnos en la cosmovisión de este pueblo milenario, donde se refiere a los anfibios como seres de fertilidad, que anuncian la lluvias, tan necesarias para el arte de la siembra; las aves, como mensajeras; el sabio jaguar, representante del liderazgo y fuerza; el cóndor, amo de los cielos; la serpiente, maestra del mundo de las plantas; los insectos, como la libélula y la mariposa, reflejan dones de profecía y, mitológicamente, el sagrado colibrí, que se convierte en el mensajero de la divinidad solar.

En el Grupo Cuatro, denominado Seres Espirituales, se plasman cinco símbolos relacionados con este tema:

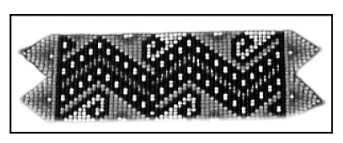

Yaco (Agua)

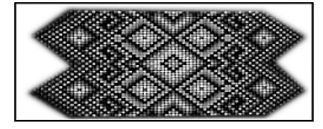

Samai (Espíritu)

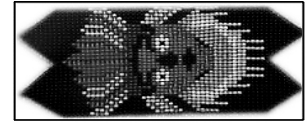

Llactu (Plumaje)

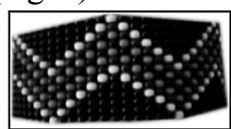

Montaña

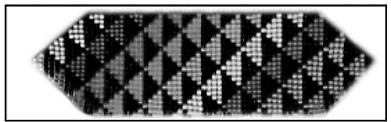

Arco iris

Figura 4. Grupo Seres Espirituales

Fuente: esta investigación

Estos símbolos se relacionan con seres protectores de la naturaleza, que se encuentran en estos lugares como huasikamas o cuidadores del entorno donde vive el pueblo inga. Estos son maestros, pero también son guerreros; por lo tanto, enseñan y reprenden; su objetivo es y será orientar y proteger.

En el Grupo Cinco, denominado Territorio, se plasman cuatro símbolos, relacionados con este tema:

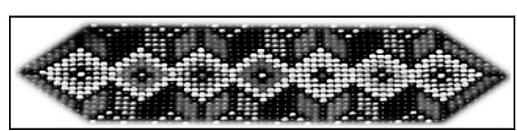

Uigsa (Rombo)

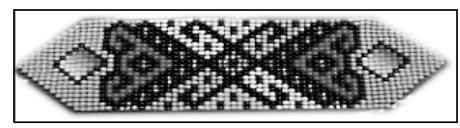

Hierros Cruzados

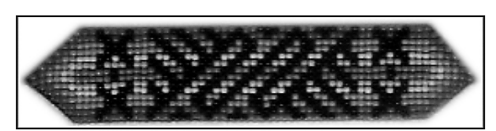

Suyu (Chagra)

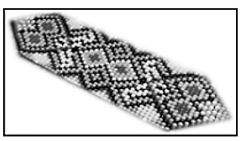

Saparro Ayllu Kutijkunapa (Canasto)

Figura 5. Grupo Territorio

Fuente: esta investigación 
En estos símbolos se pueden apreciar patrones culturales que definen la organización como una práctica de convivencia mutua y comunitaria: el rombo define los cuatro puntos de origen, la chagra es el lugar sagrado de donde se obtiene alimento, los hierros cruzados señalan en un mapa la ubicación de la comunidad y el canasto define la sabiduría de la escritura simbólica que las mamas heredan a su familia.

Por último, en el Grupo Seis, denominado Fertilidad, se plasman cuatro símbolos relacionados con este tema:

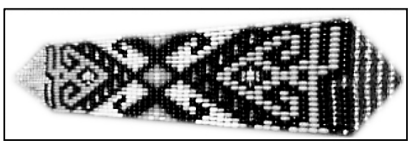

Uigsa Tujtu (Flor de Vientre)

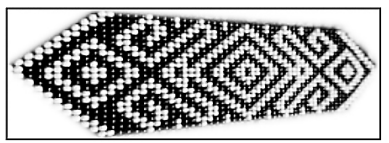

Wawa Llukai (Nacimiento)

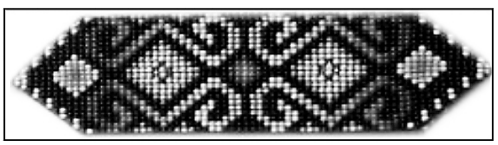

Wawa LLugsi (Dieta, Parto)

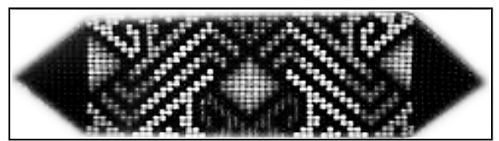

Wawata Kauai (Crecimiento)

Figura 6. Grupo Fertilidad

Fuente: esta investigación

En estos símbolos se reflejan las diferentes etapas de la vida del inga, desde su concepción, al unir su energía masculina con la femenina y formar un enlace o flor, para, luego, crecer en el vientre, formarse, nacer y crecer.

Los símbolos de las manillas presentadas fueron fotografías tomadas en esta investigación, suministradas en las entrevistas con las artesanas Aleyda Quinchua, Daysi Mavisoy, Mary Luz Quinchua, Cristóbal Córdoba (en enero de 2019) y los taitas de las etnias Siona, Cofán, en la Comunidad de Aponte y en la Maloca Kahansi Thesy, del Corregimiento de Mocondino (en junio de 2018).

Dentro de los hallazgos en este estudio, se encontró con que los símbolos trabajados por las artesanas, en las manillas, tienen características polisémicas; por lo tanto, un símbolo puede tener varias interpretaciones. Por otro lado, al tener como referencia el tejido chumbe, prenda que tiene más de 200 símbolos, sin embargo las artesanas solo utilizarían 19 de estos referentes, mientras que las visiones que producen las plantas sagradas se refieren a 11 símbolos trabajados desde estas perspectivas. 
El traslado de estos símbolos, sacados de las visiones con las plantas sagradas, a los tejidos, se realiza así: por una parte, el taita, que prepara la planta sagrada o yagé, y, por otra, según el tipo de ambihuasca o bejuco de sabiduría utilizado, cuyo espíritu se manifiesta en el ritual. Esos espíritus pueden ser el tigre, el sol, el arco iris, el colibrí, la anaconda, etc. En este sentido, la planta, según su preparación y el lugar en donde se encontró, maneja una entidad espiritual que la protege y la guarda.

Según los taitas ingas, la práctica de utilizar plantas visionarias, como el Ayahuasca (yagé), permite conectarse con la naturaleza; por ello, hay cultos al agua, el fuego, la montaña, el río, las piedras, las plantas, los animales, el arco iris, etc. La interacción chamán-naturaleza se realiza mediante el lenguaje que los curacas o chamanes manejan y que, según el taita siona Pedro Piaguaje, "es el de la misma naturaleza".

Estos saberes se representarían, también, dentro de esta escritura, sobre todo la relacionada con los símbolos de los animales, muy presentes en las manillas. Cada animal es un maestro que guarda secretos, no solo de su ecosistema, sino del mundo, entendido como una totalidad; por ello, estos animales, que ahora se representan en los símbolos y figuras, eran tan importantes en la antigüedad, se les rendían cultos y rituales, por lo que se los denominaba a ellos mismos como maestros taitas o apus.

En la antigüedad, existieron chamanes muy poderosos según los relatos de los mismos sabedores, que tuvieron una relación tan estrecha con el animal escogido por ellos, que terminaron por adoptar sus formas, mismas con las cuales ahora nos aparecerían como un animal, pero siguen guardando una conciencia humana.

Por último, los taitas solicitan a una artesana elaborar la imagen que han captado en su mente, para que lleve la experiencia a sus tejidos, o ellos mismos los elaboran.

El estudio de la simbología en los tejidos de las manillas Inga permite identificar nueve temáticas distribuidas en las siguientes categorías y contenidos tal como se muestran en la Tabla 1:

\begin{tabular}{|l|l|}
\hline \multicolumn{2}{|c|}{ Contenidos de la investigación } \\
\hline \multicolumn{1}{|c|}{ Temas } & \multicolumn{1}{c|}{ Subtemas } \\
\hline 1. CHUMBE & Símbolos \\
\hline 2. MINGAS DE PENSAMIENTO & Diálogos-Composición-Tejidos \\
\hline
\end{tabular}




\begin{tabular}{|l|l|}
\hline 3. AMBIHUASCA (YAGÉ) & Símbolos \\
\hline 4. ASTROLOGÍA & $\begin{array}{l}\text { Constelaciones: Orión, Cruz del Sur } \\
\text { Estrellas: Sirio, Sol } \\
\text { Planetas: Venus } \\
\text { Vía Láctea: Churo cósmico }\end{array}$ \\
\hline 5. PLANTAS SAGRADAS & Flor, Maíz, Ambihuasca (Yagé) \\
\hline 6. ANIMALES & $\begin{array}{l}\text { Tigre, Guacamaya } \\
\text { Águila, Serpiente } \\
\text { Colibrí, Libélula, Rana }\end{array}$ \\
\hline 7. SERES ESPIRITUALES & $\begin{array}{l}\text { Arco iris, Espíritu, Agua } \\
\text { Rio, Montaña, Plumaje }\end{array}$ \\
\hline 8. TERRITORIO & Rombo, Chagra, Hierros cruzados, Canasto \\
\hline 9. FERTILIDAD & $\begin{array}{l}\text { Vientre, Embarazo, Dieta, Parto, Nacimiento } \\
\text { Crecimiento }\end{array}$ \\
\hline
\end{tabular}

Tabla 1. Contenidos de la investigación

Fuente: registro de campo de la investigación, 2019.

\section{Propuesta Educativa Cultura y Educación: Instituto Agropecuario Inga de Aponte-Nariño}

Una vez analizados y organizados los contenidos culturales obtenidos de la investigación, correspondió diseñar, conjuntamente con los docentes, la propuesta educativa del Instituto Agropecuario Inga, de Aponte. Esta propuesta era necesaria implementar como un proceso en la formación estudiantil; por eso, fue importante realizar una socialización, que buscara una profundización de estos contenidos con la misma comunidad educativa, con el fin de fortalecer la propuesta y consolidarla en el Proyecto Educativo Institucional.

La propuesta tuvo la misión de concientizar a la comunidad educativa en formar un nuevo maestro, comprometido con los contenidos pedagógicos y el contexto social, que gira en torno a la cosmovisión de su propio mundo y una concepción orientada a la educación propia. Se trata de hacer un llamado de atención sobre los valores culturales, para que, tanto jóvenes como adultos de estas comunidades indígenas, encuentren en este campo otra alternativa de participación comunitaria, la exploraran, realizaran sus aportes y ayudaran a iniciar un diálogo de saberes entre la educación propia y la occidental. 
La comunidad educativa, al incluir en su práctica pedagógica cotidiana elementos propios de esta comunidad, permitió desarrollar su labor formativa en el intercambio de saberes con los estudiantes, lo que contribuye a sistematizar y salvaguardar los valores e identidad de esta comunidad, los conservan en sus diversas formas, para transmitirlos como parte de su historia.

Este proceso definió unidades culturales, a modo de temática, para que puedan desarrollarse con sus respectivas competencias y DBA correspondientes, dentro del Proyecto Educativo Institucional; se determinarían por grados, tiempos y clases, en los que se desarrollaran estos contenidos culturales, con la propuesta, además, de un método de evaluación a trabajar en el aula y definir cómo complementar los contenidos culturales hallados en la investigación, sobre los símbolos, con estas competencias:

- Científicas (Ciencias Sociales - Ciencias Naturales)

- Matemática

- Comunicativas

- Educación Artística.

La Institución Educativa Agropecuaria Inga ha venido trabajando una educación sin tener en cuenta las condiciones de quienes la reciben $\mathrm{y}$, en segunda instancia, en una tensión estudiantes-profesor por falta de entendimiento en el plano cultural. Esta propuesta ayuda a organizar parte del pensamiento educativo a partir de la interpretación simbólica que se hizo de los tejidos, puesto que en ellos se plasman varias temáticas, que pueden complementar las actuales áreas de estudio trabajadas desde la parte educativa.

Esto se logra en la medida en que se ha orientado a los docentes del Instituto Agropecuario Inga de Aponte con este proceso de formación educativa sobre el reconocimiento cultural, para ayudar a cualificar los procesos pedagógicos que actualmente se imparten, al mejorar las capacidades y los contextos de formación en entidades con la fortaleza de trabajar propuestas de educación propia.

Como referente teórico conceptual, esta propuesta de investigación se consultó por directivos, docentes y comunidad, para la realización de los talleres y conversatorios, en busca de guiar y profundizar en los contenidos que ayudaran al fortalecimiento y consolidación de un Plan de educación propia, dentro del proyecto educativo institucional. 
La propuesta permite, también, plantear la formación de los docentes en la organización de los contenidos culturales y simbólicos, lo que ayuda a complementar el Proyecto Educativo del Instituto Agropecuario Inga de Aponte, propuesta que se seguirá construyendo por parte de la misma comunidad educativa, ahora desde una nueva visión de maestro comprometido con los contenidos pedagógicos desde el contexto social, que gira en torno a la cosmovisión de su propio mundo.

Este trabajo trata de hacer un llamado de atención sobre los valores culturales para que, tanto jóvenes como adultos de estas comunidades indígenas, encuentren en este campo otra alternativa de participación comunitaria, la exploraran, hicieran sus aportes y ayudaran a iniciar un diálogo de saberes entre la educación propia y la occidental.

Se ha recomendado a los docentes vincular los conversatorios y/o talleres desde los contenidos de la investigación, con definición de las unidades culturales a modo de temática, para que puedan desarrollarlas con sus respectivas competencias y DBA correspondientes, de acuerdo a la materia que ellos manejaran dentro del Proyecto Educativo Institucional.

\subsection{Objetivos}

El objetivo general de la propuesta es comprender los aspectos simbólicos de la cultura Inga que se encuentran presentes en los tejidos de las manillas y son importantes para fortalecer el Proyecto Educativo Institucional del Instituto Agropecuario de Aponte. Para ello de manera específica se planteó:

- Dar unos fundamentos generales de la Cultura Inga en la Educación Propia, por parte de la comunidad educativa.

- Análisis de los contenidos culturales y simbólicos de las manillas Inga.

- Orientar la construcción de una propuesta para la formación de los docentes en la organización de los contenidos culturales y simbólicos que complementen el Proyecto Educativo del Instituto Agropecuario Inga de Aponte. 


\subsection{Justificación}

Una propuesta educativa con enfoque basado en los contenidos de interpretación simbólica de la cultura inga es responsabilidad de las directivas y docentes, que deben proporcionar a la Institución Educativa una educación que les permita capacitar a los docentes desde una alternativa educativa, cuya interpretación de la realidad abre escenarios hacia contextos pedagógicos que posibiliten la educación propia en estas comunidades indígenas.

Un buen número de profesores no son del resguardo inga de Aponte y desconocen la historia del pueblo inga. Esto no permite implementar una educación con sentido para los estudiantes de origen indígena; la tradición de implementar procesos educativos sin pertinencia cultural o socioeconómica, más que dar solución y procurar desarrollo de las comunidades, tiende a generar tensiones y conflictos.

Trabajar estas expresiones culturales desde los ámbitos de la investigación es una alternativa que contribuye a sistematizar y salvaguardar los valores e identidad de esta comunidad, la conservan en sus diversas formas, para transmitirla como parte de su historia, para que los resultados sirvieran como un aporte a la actividad educativa del Proyecto Educativo Institucional.

Se pretende generar procesos hermenéuticos, que ayuden a los docentes de la Institución Educativa Agropecuaria Inga de Aponte a incluir en su práctica pedagógica cotidiana elementos propios, que permitan desarrollar su labor formativa en el intercambio de saberes con los estudiantes; en este sentido, fomentar la investigación desde las aulas fortalece sus costumbres, sus prácticas culturales y, también, la apropiación de nuevos procesos como pueblo, desde el reconocimiento y respeto de otras etnias.

Los conversatorios y/o talleres permiten iniciar esa construcción de una propuesta educativa orientada desde los contenidos culturales, guiados desde este trabajo de investigación, que vinculan a docentes, estudiantes, padres de familia y comunidad en general a nuevos espacios de integración y formación social.

\subsection{Acciones para el desarrollo de la Propuesta Educativa}

En el desarrollo de esta propuesta educativa, se propone como metodología trabajar cuatro conversatorios y un taller.

En el primer y segundo conversatorio, denominados Fundamentos generales de la Cultura inga en la educación propia, se estudia el marco 
contextual, páginas 27-42 de la Investigación Iuiay Kamsaykug Tantalli (Tejido del pensamiento, una propuesta inga de educación propia), para realizar una exposición de los conocimientos sobre la historia del pueblo inga, como complementariedad de conceptos, donde participan los docentes de la comunidad educativa, al igual que taitas mayores de la comunidad, artesanas y estudiantes. La duración de este taller es de dos semanas, con una totalidad de ocho horas trabajadas.

En el tercer y cuarto conversatorio, se estudia el marco contextual y el capítulo dos, denominado: Lo que dicen las manillas, páginas 45-110 de la Investigación Iuiay Kamsaykug Tantalli (Tejido del pensamiento, una propuesta inga de educación propia); en este espacio, se realiza un análisis de los contenidos culturales y simbólicos de las manillas Inga, para lograr un diálogo con los docentes de la institución, los mayores de la comunidad, las artesanas y los estudiantes. La duración de este taller es de cuatro horas semanales, con una totalidad de 16 horas trabajadas.

Por último, se realiza un Taller, que busca orientar la construcción de una propuesta para la formación de los docentes en la organización de los contenidos culturales y simbólicos, que complementen el Proyecto Educativo del Instituto Agropecuario Inga de Aponte; para ello, se estudian las conclusiones de este trabajo de investigación y el Capítulo 3, denominado "La propuesta educativa, páginas 111-119 del Proyecto: Iuiay Kamsaykug Tantalli (Tejido del pensamiento, una propuesta inga de educación propia). Durante este intercambio de saberes, se exponen los alcances pedagógicos de esta propuesta educativa en la comunidad inga, con participación activa de artesanas, taitas y docentes de la comunidad, en un constante diálogo y retroalimentación constante.

Este proceso permite definir, por parte de la comunidad de aprendizaje, cuáles serían los símbolos que se podrían implementar como temas de estudio, dentro de las competencias educativas de aprendizaje, en el actual currículo institucional. Este taller dura dos meses, en una totalidad de 48 horas trabajadas.

\subsection{Competencias incluidas dentro de los Contenidos Culturales trabajados}

Se definen unidades culturales a modo de temática, para que puedan desarrollar los docentes, teniendo en cuenta la disciplina que cada cual lidera dentro del Proyecto Educativo Institucional (PEI). 
- Científicas (Ciencias Sociales - Ciencias Naturales)

- Matemática

- Comunicativas

- Educación Artística

\subsection{Actividad final}

Socialización, a los profesores, de la propuesta educativa de cada área pedagógica, teniendo en cuenta los referentes simbólicos.

Estrategias metodológicas para los Conversatorios y/o Talleres

- Las clases tienen enfoque de seminario, donde se le facilita una lectura previa al auditorio y, con base en ella, se realiza un debate.

- Cada sesión se complementará con una sesión práctica de trabajo independiente.

- Lectura de artículos relacionados con temas abiertos en investigación.

- Exposiciones de temas avanzados por parte de los asistentes.

- Presentaciones de máximo 45 minutos.

- Presentaciones breves, con uso de herramientas tecnológicas.

- Enviar por correo la presentación y los ejemplos respectivos.

- Trabajar las temáticas en equipo.

- Es muy importante que los responsables del evento hubieran leído los documentos recomendados para los conversatorios y/o talleres.

Recursos Recomendados para los Conversatorios y/o Talleres

- Aula con video beam.

- Acceso a Internet.

- Tablero - Marcadores - Resma de papel - Colores - Cartulina.

- Otros, que cada docente determine, según la necesidad pedagógica.

Bibliografía de la Propuesta Educativa

- Documentos de la investigación entregados por el docente.

- Estándares Básicos de Competencias, Ministerio de Educación Nacional, 2002.

- Derechos Básicos de Aprendizaje, 2018. 


\section{Conclusiones}

La investigación, como ya se mencionó, ha permitido conocer unos contenidos relacionados con la simbología inga, que ayudan a categorizar unos temas y subtemas, que fortalecen los contenidos en las distintas áreas educativas: la competencia científica, donde se incluyen las ciencias sociales y las ciencias naturales, junto a las competencia matemática, comunicativa y educación artística.

Por su parte, estas áreas cumplen de manera clara unos determinados objetivos y derechos básicos de aprendizaje; cada competencia puede desarrollar y ampliar mucho más el conocimiento de una determinada área, orientada por los lineamientos que el Ministerio de Educación ha desarrollado en estos últimos años y que, al incluir la temática de la simbología, se observa un mayor aprovechamiento del currículo escolar, orientado desde el componente de educación propia, en esta institución educativa indígena.

La propuesta permite plantear la formación de los docentes en la organización de los contenidos culturales y simbólicos, lo que ayuda a complementar el Proyecto Educativo del Instituto Agropecuario Inga de Aponte, propuesta que se tendrá que seguir construyendo por parte de la misma comunidad educativa, desde una nueva visión de la investigación educativa, que tenga presentes los contenidos pedagógicos desde el contexto social, que gira en torno a la cosmovisión de su propio mundo.

Se observa, también, un desinterés por parte de los historiadores de profundizar y restituir el legado histórico indígena; por ello, lo esencial de inculcar desde las aulas estas propuestas en los estudiantes, que permiten formular debates que orienten en la necesidad de incluir la parte tanto investigativa como de pensamiento crítico en su formación y desarrollo ciudadano, en busca de ser cada vez más conscientes de su protagonismo social en un país pluriétnico y multicultural, donde el Estado tiene la obligación de garantizar sus derechos y reconocer su identidad cultural e histórica.

Por último, este trabajo permite establecer un reconocimiento a los pueblos indígenas, gestores en épocas milenarias de siete importantes procesos, que originaron su expresión simbólica, que en esta investigación se ha resaltado. Dentro de estos procesos, se destacarían: primeramente, la escritura, para llegar a esta conclusión: los símbolos presentes, tanto 
en los tejidos como en las manillas Inga, forman parte de una escritura muy antigua, conocida por los originarios como Togapu Quellga; los investigadores actuales la han calificado como escritura nostrática o el código Wira Cocha, del que surgirían los cinco tipos de escritura preamericana, como el sistema Uri, Cuneiforme, Jeroglífico, Pictográfico y fonético, que, a la vez, darían forma al actual Runa Simi, un sistema de escritura presente en los tejidos actuales, que es una recopilación o resumen de estas ideas complejas de representar a través de símbolos, nuestra interpretación del mundo.

En segundo término, se resalta, dentro de los procesos de conocimiento andino, la ciencia astronómica, sobre la cual hay mucha evidencia arquitectónica, mitológica y simbólica; desde ese punto de vista, el estudio de las constelaciones, estrellas, planetas y calendarios sería un reflejo claro de esta sabiduría. Precisamente, el estudio de los astros permite medir ciclos, días, meses y años, para convertirse en una ciencia exacta, desde donde el tiempo se define, para surgir la matemática conocida, en nombre originario, como Yapay Camai; de ella se derivan otros conocimientos de operaciones en la resolución de problemas con números, espacios y medidas, para ser: Quilkanachancamay (álgebra), Pachay Camai (Física), Suyutupucamai (Geometría), Quinsamanchayay camai (Trigonometría), y la arquitectura como el cuarto proceso, conocida en nombre originario como Coyuyachai, que se representaría simbólicamente en cuatro referentes: el rombo, la chacana, el churo y la estrella de ocho puntas.

El rombo, conocido como teolt, o los cuatro puntos cardinales, se llegó a utilizar como un sistema de medida de espacios físicos; la chacana, por su parte, se utilizaba para medir precisamente el tiempo, es decir era un calendario; el churo se refiere a los ciclos naturales, regidos, también, por secuencias matemáticas, conocidas como la secuencia Fibonacci, desde la cual todos los organismos compartiríamos las medidas de este patrón y, por último, la estrella de ocho puntas, que tendría la función de ser un reloj astronómico. Se resaltan, en este tema, los quipus, un sistema de almacenamiento de información; la yapana, una calculadora exacta, y la utilización del Katari o número pi, para expresar matemáticamente formas y medidas.

El quinto y sexto proceso en consolidarse es la agricultura, seguida por la hidráulica, actividades mutuamente relacionadas con el desarrollo de la civilización, cuyos avances se muestran en el tratamiento de la tierra por 
parte de las etnias indígenas milenarias, que supieron entender y manejar el entorno en beneficio tanto de sus intereses como de sus necesidades, lo que testifican los alimentos naturales de consumo y los acueductos antiguos, que aún funcionan.

El último proceso sería la filosofía, conocida por los pueblos quechuas, del que forman parte los ingas, como el Suma Kausay (el buen vivir), resumido en esa armonía que tiene el ser humano consigo mismo, con los demás y con el entorno; esta es tanto una filosofía de vida, como un tipo de mandamientos: no robar, no ser mentiroso, no ser perezoso y ser digno, se convierten en unas normas de comportamiento, que hacen del indígena un ser íntegro y único, del cual tenemos muchas cosas que aprender.

Todos estos conocimientos muestran la importancia de haber trabajado esta investigación con la comunidad indígena inga, por lo cual es muy necesario vincular estos contenidos en las instituciones educativas de las comunidades indígenas $\mathrm{y}$, posteriormente, en las de nuestra sociedad urbana, que tanto les hace falta concientizarse sobre esa necesidad de cooperativismo entre nosotros y respeto por nuestra propia naturaleza, para lograr cambios, en mejora de nuevas visiones de desarrollo y, también, de nuevas reflexiones sobre el pensamiento histórico.

Se recomienda a los docentes continuar trabajando los contenidos de la investigación desarrollada, definir más unidades culturales a modo de temática, para que puedan desarrollarlas con sus respectivas competencias y DBA correspondientes, de acuerdo a la materia que ellos manejarán dentro del Proyecto Educativo Institucional.

Por la complejidad de la temática, que exige un análisis y comprensión tanto detallada como analítica, es fundamental estudiar los contenidos de la investigación con estudiantes de nivel de secundaria, tanto para un mejor aprovechamiento como para la asimilación de los conceptos; sin embargo, se podría trabajar desde las competencias de educación artística y matemática con los estudiantes de los niveles de primaria.

Por otra parte, es necesario establecer una revisión sobre los procesos históricos del cómo se han dado a conocer, por parte de docentes e historiadores, los pueblos antiguos indígenas de América; es importante replantear estos enfoques de información, para fortalecer los conocimientos sobre estas culturas ya extintas o por extinguirse.

Se necesitan docentes con una nueva conciencia histórica sobre nuestro pasado; esto implica efectuar una actualización en las nuevas 
investigaciones y teorías desarrolladas a lo largo de estos años, para construir un espacio de reflexión, donde esta propuesta de investigación permita dar unos referentes importantes a tener en cuenta, desde un sentido mucho más pedagógico y constructor de esas nuevas visiones.

Es muy importante seguir posicionando los conocimientos de estos pueblos ancestrales a través de estas propuestas educativas; lastimosamente, estos pueblos han venido siendo maltratados por el Estado y su sistema depredador, que desconoce los aportes de su historia y, más aún, esa convivencia como armonía del ser humano y del espacio que, como filosofía, el indígena da a conocer desde su interacción; por ello, todos estos procesos son fundamentales para ayudar a construir una sociedad más pluriétnica y democrática, con todos sus actores sociales.

\section{Referencias}

Ágreda, Antonia. "La influencia del español en el idioma inga". Tesis de grado, Universidad Distrital Francisco José de Caldas, 1993. Mec.

Aramayo Canedo, Lucia. "El espacio vivido andino en imágenes paceñas y cuando Sara Chura despierte". Recial, vol. 7. No. 9, (2016): 1-17.

Arango, Raúl. Los pueblos indígenas de Colombia en el umbral del nuevo milenio. Bogotá: DNP, 2004. Bogotá.

Beciez, David. Etnografía educativa. México: McGraw-Hill, 2009.

Briones, Guillermo. Investigación cualitativa. Bogotá: Arfo Editores, 1993.

Buck, Frizt. Un magnifico ejemplo de inscripciones calendáricas. Lima, Perú: Editorial Aguirre, 1939.

Chirán Caipe, Rosa Alba y Burbano Hernández, Marleny. (2013). “La dualidad andina del pueblo Pasto, principio filosófico ancestral inmerso en el tejido de la guanga y la espiritualidad”. Plumilla educativa, vol. 11. No. 1 (2013): 136-156.

Delgado Jacanamejoy, Doris. "Fortalecimiento de la Gobernabilidad", http:// www.ozip.org.co/pueblos/inga/documen tos/pita_kanchi.pdf 
Durán López, María Elisa. "Sumak Kawsay o Buen Vivir, desde la cosmovisión andina hacia la ética de la sustentabilidad”. Pensamiento Actual, vol. 10. No. 14, (2010): 51-61.

Friedemann, Nina S. de y Arocha, Jaime. (1985). "Del jaguar y la anaconda". En: Herederos del jaguar y la anaconda. Bogotá: Valencia editores, 1985. 17-80.

Galdames Rosas, Luis y Díaz Araya, Alberto. "Piedra en la piedra, ¿el hombre dónde estuvo? Percepción y significado de la piedra en la geografía sagrada de las sociedades andinas". Runa, vol. 36. No. 2, (2015): 5-23.

García Escudero, María del Carmen. "Estudio sobre el nacimiento y la composición anímica del hombre en la cosmovisión prehispánica centroandina”. Anales del Museo de América. No. 19, (2011): 211-232.

García Gavidia, Nelly. "El uso de símbolos indígenas en la invención de la identidad nacional". Opción, vol. 19. No. 40, (2003): 9-34.

Geertz, Clifford. La interpretación de las culturas. Barcelona: Gedisa, 1973.

Gobernación del Putumayo y Secretaría de Planeación. "Documento de memorias Taller Plan de Desarrollo indígena Inga-Kamentsá del Valle de Sibundoy, evento realizado en marzo 6, 7 y 8 de 2001 '.

González-Varas, Ignacio. Conservación del Patrimonio Cultural: teoría, historia, principios y normas. Madrid: Cátedra, 2000.

Granda Paz, Osvaldo. Tejedoras de guango. Textiles en los Andes nariñenses. Pasto: Universidad de Nariño, Facultad de Artes, 1995-1996.

Irrazával, Diego. "El desafío intercultural y la teología andina". Polis. No. 18, (2007), https://journals.openedition. org/polis/4068:

Jacanamijoy, Benjamín. "El arte de contar y pintar la propia historia". Mundo Amazónico. No. 5, (2014): 211-219.

Kreimer, Elizabeth. "Curar es el arte de decir las cosas". En: Lenguajes y palabras chamánicas. Chamanismo y simbolismo onírico en el pueblo achuar, coordinado por Patrice Bidou y Michel Perrin. Quito: Abya-Yala, 2000. 151-183. 
Gómez Hernández, Ivonne Rocío. “Las Formas de Tejer la Vida: ¿Cómo vivir una experiencia Inga a través del diseño, en el museo de trajes regionales?". Trabajo de Grado, Pontificia Universidad Javeriana, 2012.

Illicachi Guzñay, Juan. "Desarrollo, educación y cosmovisión: una mirada desde la cosmovisión andina”. Universitas, vol. 12. No. 21, (2014): 17-32.

Jacanamijoy, Benjamín. El Chumbe Inga. Una forma artística de percepción del mundo. Bogotá: Ankla editores, 2017.

Jacanamijoy Chasoy, Edgar y Bastidas Jacanamijoy, Lizbeth. "Estudio sobre los simbolismos en las manifestaciones artísticas visuales de la comunidad indígena Inga de Santiago, Putumayo". Revista Educación y Pedagogía. No. 49, (2007): 173-183.

Jara, Victoria de la (1964). La escritura peruana y los vocabularios quechuas antiguos. Lima: Editorial Futura, 1964.

"Kamentsa: antiguos sueños, nuevas interpretaciones". En: Lenguaje creativo de etnias indígenas de Colombia, autor Cecilia Duque Duque. Medellín: Suramericana, 2012, http://artesaniasdecolombia.com.co/PortalAC/ Noticia/kamen tsa-antiguos-suenos-nuevas-interpretaciones_5531

Koch, Rudolph. El libro de los simbolos. Madrid: Manakel editorial, 2010.

Lenguas de Colombia. Página web institucional, http://www.lenguasdecolombia. gov.co

Levinsohn, Stephen H. "Inga". En: Aspectos de cultura material de grupos étnicos de Colombia 1, editado por Ministerio de Gobierno. Bogotá: ILV/ Ministerio de Gobierno, 1973. 279-291.

Martínez Sarasola, Carlos. Identidad, cosmovisión y espiritualidad en los pueblos indigenas. Buenos Aires, Biblos, 2010.

Ministerio de Cultura. “Ingas, el pueblo viajero”. Bogotá: Ministerio de Cultura, 2010.

Observatorio del Programa Presidencial de DH y DIH. "Diagnóstico de la situación del pueblo indígena Inga", http://2014.derechoshumanos.gov. co/Observatorio/Documents/2010/DiagnosticoIndigenas/Diagnostico_ KAMS\%C3\%81.pdf 
Pinzón, Carlos Ernesto y Garay, Gloria. "Inga y Kamsá”. En: Geografia Humana de Colombia, coordinado por François Correa R. Tomo 4. Vol. 3. Bogotá: Instituto Colombiano de Cultura Hispánica, 1996, http://babel.banrep cultural.org/digital/collection/p17054coll10/id/2824

Pueblos Indígenas Ingas (Departamento del Putumayo). "Proyecto: Proceso de construcción del plan integral de vida del pueblo inga en el Departamento del Putumayo". Mocoa-Putumayo: 2003.

Quiguanás Cuetia, Abraham. "Los tejidos propios: Simbología y pensamiento del pueblo Nasa". Trabajo de grado, Universidad del Cauca, 2011.

Rivadeneiva, Severo y Zubritski, Yuri. "Algunas observaciones de campo en torno a un grupo quechua mitimae (inga putumayense)". Sarance, vol. 3. No. 1. (1977): 58-62.

Rodman, Amy Oakland y Fernández, Arabel. "Los tejidos huari y tiwanaku: comparaciones y contextos". Boletín de Arqueología PUCP. No. 4 (2000): 119-130.

Rozo Vidal, Antonio. "El carnavalito entre los indígenas inganos de Yunguillo". Revista Colombiana de Folklor, vol. 2. No. 6, (1961): 143-152.

Seibold, Katharine. "Oraciones tejidas: misaq'epi y el despacho a la pachamama". Cuadernos. No. 17, (2001): 445-454.

Spradley, James P. Participant observation. Nueva York, NY: Holt, Rinehart y Winston, 1980.

Taylor, Steve J. y Bogdan, Robert. Introducción a los métodos cualitativos de investigación. Buenos Aires: Paidós, 1986.

Tandioy Ch., Domingo y Levinsohn, Stephen H. "Términos de parentesco inga". Artículos en lingüística y campos afines. No. 4 (1978): 77-81. Mec.

Terrón de Bellomo, Herminia. "El motivo del tejido en los relatos orales de Jujuy: un rasgo identitario de la cultura andina". En The last Incas: Social change as reflected in the textiles of Choquecancha. Ann Arbor: Indiana University, 1990.

Urrea Giraldo, Fernando. "Remedios botánicos y modelo etnomédico en el curanderismo Ingano, Kamsa". En: Memorias V Congreso Nacional de 
Antropología, Vol. 1.: Curanderismo en la Colombia Contemporánea. Bogotá: Pontificia Universidad Javeriana, (1992). 121-128.

Urrea, Fernando y Puerto, Fernando. "La población Inga residente en 7 ciudades colombianas. Un caso de pobreza". Boletín de estadística DANE. No. 466 (1992): 170-209.

"Usos y saberes locales sobre biodiversidad. Inga", http://www.siac.net.co/ yoscua/bin/view/Principal/Inga.

Ulloa Sanmiguel, Alejandro y Carvajal Barrios, Giovanna. Teoría del texto y tipología discursiva. Cali: Universidad del Valle, 2004.

Valcárcel, Luis E. Historia del Perú antiguo. Lima: Editorial Juan Mejía Baca, 1971.

Vega Centeno, Teobaldo Ugarte. "El desnudo en la escultura inca. Análisis y planteamientos sobre una teoría de proporcionalidad escultórica andina". Lex, vol. 11. No. 12, (1991): 353-376.

Walsh, Catherine. Interculturalidad, conocimientos y decolonialidad. México: Editorial Encuentros Multidisciplinarios de Educación Intercultural, 2005.

\section{Citar este artículo}

Muñoz Burbano, Francisco Andrés. "Las manillas como tejido del pensamiento del pueblo Inga". Revista Historia de la Educación Colombiana. Vol. 24 No 24 (2020):155-180

DOI: https://doi.org/10.22267/rhec. 202424.77 